\title{
Oral mucosa: benignity, ulcerations, erosions, papillae
}

\begin{abstract}
Cognizance of the conventional superficial non malignant oral lesions e.g. candidiasis, herpes labialis, recurrent apthous stomatitis, erythema migrans, hairy tongue, lichen planus necessitate a comprehensive history and an oral exam for identification, comprising of parameters like size, location, morphology, colour, pain, duration etc.
\end{abstract}

Volume 6 Issue 2 - 2018

\author{
Anubha Bajaj \\ University Panjab University, India
}

Correspondence: Anubha Bajaj, Designation Consultant Pathologist, University Panjab University, Chandigarh, India, Tel +0091।I 25II 7399;009198 II69 3956; Email

anubha.bajaj@gmail.com, anubha.bajaj@yahoo.com

Received: January 09, 2018 | Published: March 05, 2018

\section{Oral candidiasis}

A typical, localized infection with a potential to evolve into systemic candidiasis. Observable patterns are psuedomembranous candidiasis, thrush, median rhomboid glossitis, erythematous candidiasis, perleche' or angular cheilitis. Systemic infection is delineated in autoimmune deficiency syndrome, immunosuppressive therapy, hospitalization, diabetes mellitus, neutropenia, malignancy, organ transplantation and prematurity. Mild infections of the oral cavity, oropharynx and skin elucidate infection in the infancy (Candida diaper dermatitis). Chronic mucocutaneous candidiasis is affiliated with endocrine disorders such as hyperparathyroidism, ${ }^{1}$ hypoadrenalism, hypothyroidism, and diabetes mellitus.

\section{Herpes labialis}

Asymptomatic, primary infection with herpes simplex virus, developing at an early age. Herpes simplex virus may exhibit latency in the trigeminal ganglion and reactivate to herpes labialis (cold sores) subsequent to the primary oral contamination. Reactivation is incited by ultraviolet light, trauma, fatigue, stress and menstruation. A restricted cluster of small vesicles on the vermilion border of the lip/adjacent skin is manifest. They rupture, ulcerate, and crust in 48 hours and improve spontaneously in $7-10$ days. ${ }^{2-5}$ Gross gingival modification is detected. In symptomatic primary infection, the oral vesicles demonstrate erythema and ulceration.

\section{Recurrent apthous stomatitis}

Canker Sores is an established painful, oral ulcerative lesion of an obscure aetiology, frequenting childhood and adolescent females. The mucosal disorder is a $\mathrm{T}$ cell mediated immunological reaction. Adjunctive conditions are nutritional deficiencies (vitamin B12, folate, iron), inflammatory bowel disease, coeliac disease, behcet's syndrome and human immunodeficiency viruses. Solitary or multiple painful ulcers, covered with whitish yellow pseudomembrane, surrounded by an erythematous halo occur with a cyclical pattern. Apthous stomatitis affects non-keratinizing mucosa (labial, buccal, ventral tongue). Clinical Categories elucidated are: major, minor, herpetiform. Minor ulcers are 3 to $10 \mathrm{~mm}$ and recover in 1 to 2 weeks..$^{6-8}$ Major ulcers are more than $1 \mathrm{~cm}$ and heal in approximately 6 weeks, localized in detached buccal and labial mucosa. Herpetic ulcers are restricted to the gingival and recuperate within 7 days. The condition needs to be distinguished from Sweet's syndrome, MAGIC syndrome, PFAPA syndrome, cyclic neutropenia.

\section{Erythema migrants}

Also ratified as benign migratory glossitis, is characterized by multiple zones of distinct red and white (geographic tongue). Typically prevalent in the females and children, the oral inflammatory condition of obscure aetiology. Atopy and psoriaisis coexist. Atrophy of the filiform papillae, central erythema with yellow to white raised perimeter arise in the lesions of the tongue. Exacerbations and a migrating pattern is demonstrable in what is a chiefly asymptomatic lesion. Taste is not affected. The precise, diagnostic manifestation needs to be distinguished from oral candida infection (Figure 1).

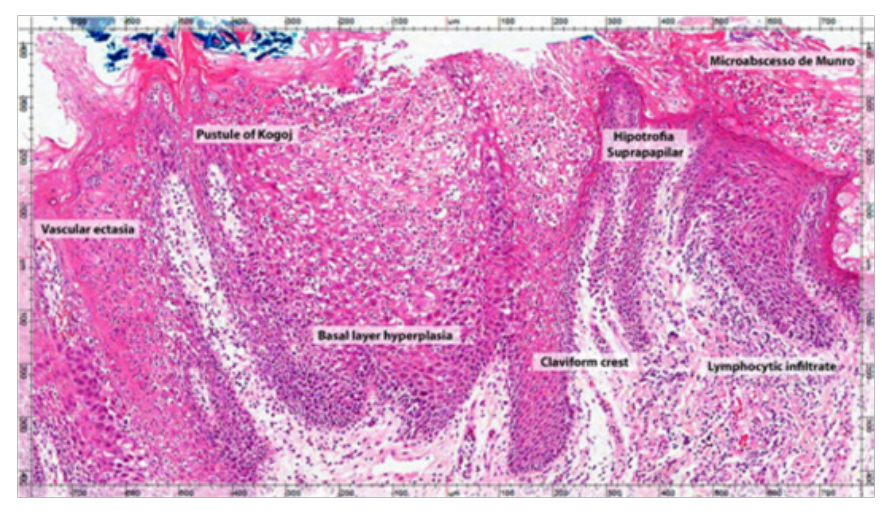

Figure I Geographic Tongue.

\section{Hairy tongue}

It is discerned by elongated, hypertrophic filiform papillae on the dorsal tongue with hair like manifestations due to inadequate desquamation or increased keratinization of the papillae. The papillae usually $1 \mathrm{~mm}$ in length, elongate to $12 \mathrm{~mm}$. Women and children are predominantly affected. Heavy smoking, poor oral hygiene, oxidizing 
mouth washes, candida albicans, certain medications are corroborating factors. Though perceived as Black hairy tongue, the condition demonstrates a black, brown or yellow discoloration, subject to the ingested foods, tobacco, tea and coffee. Metallic taste or gagging is associated Debris entrenched between elongated papillae accounts for the halitosis. Oral hairy leukoplakia must be distinguished from black hairy tongue. Bilateral, vertical white striations usually occur on the lateral tongue (Figure 2).

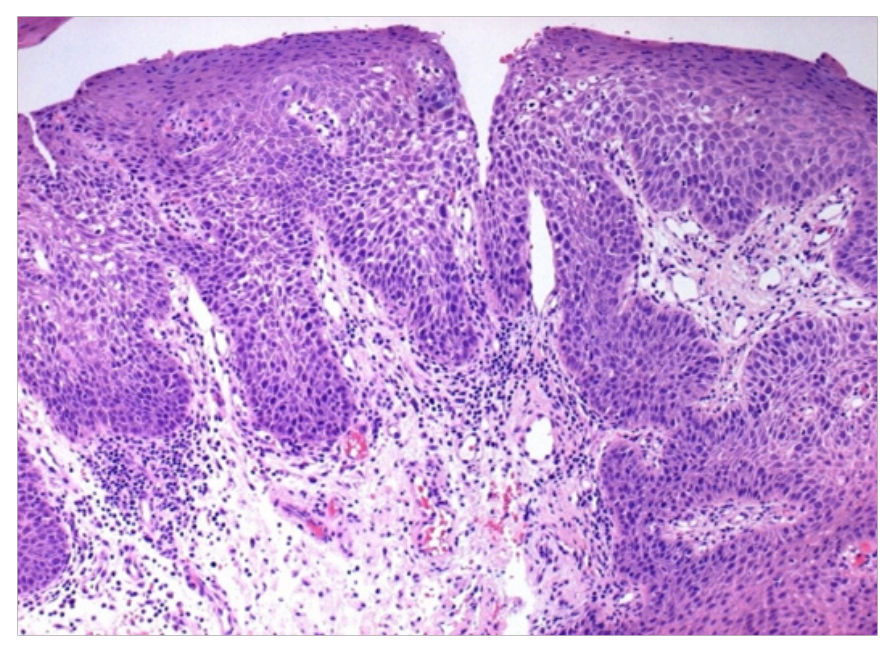

Figure 2 Psoriform Epidermal Hypertrophy.

\section{Lichen planus}

Adiffuse chronic inflammatory mucocutaneous disease categorized by cutaneous lesions which are multiple, pruritic, purplish, polygonal papules. Extensor surface dermatological lesions manifest minimal inflammation of indeterminate aetiology occurring in 1 to $2 \%$ of the adults. Immune mediation by $\mathrm{CD} 8+$ cytotoxic $\mathrm{T}$ cells incites epithelial apoptosis. The condition occurs at all ages, peaking at 40 years of age. Female to male ratio is 1.4: 1.Two dominant clinical forms prevail: reticular and erosive. The reticular configuration is bilateral and asymptomatic with white lacy striations (Wickham's Striate) or papules on the posterior buccal mucosa, similar to leukoplakia. The erosive form elucidates erythema, tenderness and painful ulcers with peripheral, white radiating striae. Desquamative gingivitis displays generalized erythema and gingival ulceration. Reticular lesions can be clinically classified and standardized. A diagnostic biopsy is required for ambiguous, non specific lesions. Oral lichen planus can be detected with oral cancer. Periodic follow-up of patients with tissue evaluation is essential for isolated lichen planus to exclude premalignant and malignant scenarios. Tobacco /Alcohol intake should be curbed. The condition needs to be differentiated from drug reactions, graft versus host disease, oral contact stomatitis due to dental materials (amalgam) Erosive form require demarcation from systemic lupus erythromatosus, chronic ulcerative colitis. Gingival lesions resemble pemphigus vulgaris and pemphigoid. Immunofluorescence on tissue examination is diagnostic.

\section{Traumatic ulcer}

A designated localized area of discontinuity in the surface epithelium of diverse shapes and sizes. Buccal mucosa, tongue and lips exhibit an erythema concealed by a variable, dense, yellow membrane. The ulcerated edge can pile up and exhibits hyperkeratosis. Simple traumatic ulcer can arise from concurring irritation, mechanical damage from mastication, toothbrush injury, malocclusion, broken down or sharp teeth, placement of restorations, prosthesis irritation, injury during sleep, ${ }^{9}$ thermal, chemical, electrical burns. Histopathology shows a definitive ulcer with elevated, ulcerated and indurated margins called Traumatic Ulcerative Granuloma with Stromal Eosinophilia (TUGSE), which may be analogous to a squamous cell carcinoma. Deep inflammation of the tongue resolves gradually and a biopsy is prescribed. Squamous cell carcinoma, Riga Fede disease, TUGSE, Ulcerative mucosal disease are conditions to be discriminated from. ${ }^{10}$

\section{Inflammatory papillary hyperplasia}

It is an asymptomatic, reactive tissue generated on the hard palate beneath an ill fitting dental prosthesis, with continual use of prosthesis, poor oral hygiene and mouth breathers, occasionally seen on a mandibular edentulous ridge or the surface of an epulis. The mucosa is erythematous and pebbly. ${ }^{11}$ Candida infection can inculcate the lesion. Keratosis follicularis (Darier's disease) is a condition to be distinguished (Tables 1).

Table I Prosaic benign oral predicaments

\begin{tabular}{|c|c|c|}
\hline Candidiasis & $\begin{array}{l}\text { Pseudomembrane, White Plaques, Red } \\
\text { Macules }\end{array}$ & $\begin{array}{l}\text { Exfoliative Cytology, Biopsy or Culture, Periodic } \\
\text { Acid Schiff or Potassium Hydroxide Evaluation }\end{array}$ \\
\hline Recurrent Herpes Labialis & $\begin{array}{l}\text { Congregation of vesicles which ulcerate and crust. A } \\
\text { prodrome }\end{array}$ & Activated by ultraviolet light, trauma, fatigue and stress. \\
\hline Recurrent Apthous Stomatitis & \multicolumn{2}{|c|}{ Yellow white pseudomembrane, ulceration with peripheral erythema } \\
\hline Erythema Migrans & \multicolumn{2}{|c|}{ Migratory erythematous lesions with elevated, yellow borders. On tongue. } \\
\hline Lichen Planus & $\begin{array}{l}\text { Reticular: white, lacy striae. Erosive: erythematic and } \\
\text { ulcers with peripheral radiating striae. Gingival ulcers. }\end{array}$ & $\begin{array}{l}\text { Buccal lesions are chiefly reticular. Tongue and gingival } \\
\text { involvement. }\end{array}$ \\
\hline Hairy Tongue & Elongated , Filiform papillae & $\begin{array}{l}\text { Induced by poor oral hygiene, smoking, antibiotics \& } \\
\text { psychotropics. }\end{array}$ \\
\hline Traumatic Ulcer & $\begin{array}{l}\text { Erythematous Ulcer with yellow membrane and } \\
\text { hyperkeratotic edge }\end{array}$ & TUGSE: \\
\hline Inflammatory Papillary Hyperplasia & Reactive ulceration on the hard palate & Caused by III fitting prosthesis and Medication Burns \\
\hline Epulis Fissuratum & Exuberant, ulcerated, hyperkeratotic tissue. & \\
\hline Dentrifice Related Sloughing & Ulceration with stringy mucosa and keratin. & Generated by products of dentrifice \\
\hline
\end{tabular}




\section{Epulis fissuratum (inflammatory fibrous hyperplasia)}

Lesions comprise of folds of hyperplastic tissue adjacent to the complete or partial denture set, often in the maxillary vestibule. The inflamed tissue is firm, fibrotic, and exuberant and can resemble a pyogenic granuloma. The precise lesion extends from less than $1 \mathrm{~cm}$ to the entire length of the vestibule. Ill-fitting dentures in the geriatric population and hyperplasia of the oral mucosa are determinants. Contact stomatitis due to artificial cinnamon flavouring/ medication burns in ice cream, candy, gum, mints, toothpastes, mouthwashes and floss requires differentiation. Prominent gingival enlargement, oedema with sloughed up superficial erythematous epithelium is encountered. Gingival reaction is more localized, occurring in buccal mucosa and lateral tongue. The lesions have an erythematous base but a white covering of hyperkeratosis and extension to the dorsal tongue. Cinnamon contact stomatitis is proved to be an intra oral contact allergy. Clinical appearance, clinical improvement with cessation of the offending agent are typical aspects along with attributes of leukoplakia, hairy leukoplakia and lichenoid reaction. ${ }^{12}$

\section{Dentifrice related sloughing}

Of the oral mucosa are an anomalous discovery and the caused by numerous dentifrices. Lesions are asymptomatic or erythematous and accompanied by a burning sensation. White, stringy mucosal expanse with sloughed, superficial keratin is observed. Food additives, flavoring agents, abrasives, ${ }^{13}$ detergents, tartar control agents, tetra sodium and tetra potassium pyrophosphate and fluorides instill reactive changes in the oral mucosa. The susceptible population can consider the modification of dentifrice, removal/alteration of chemicals. Elderly population, continued application and hypersensitivity to the blend require assessment. Response to other oral chemicals should be analyzed.

\section{Acknowledgement}

None.

\section{Conflict of interest}

Author declares that there is no conflict of interest.

\section{References}

1. Wand C, Gonsalves. Common Oral Lesions: Part I, Superficial Mucosal Lesions. Am Fam Physician. 2007;75(4):501-506.

2. Ana Cecília V, Patrick A, Paula Y, et al. Clinical, epidemiological and therapeutic profile of patients with brachioradial pruritus in a reference service in dermatology. An Bras Dermatol. 2016;91(04).

3. Image 2 courtesy pathology outlines.

4. Diagnosis and Management of Oral Lesions and Conditions. A Resource Handbook for the Clinician, Intech. India; 2014.

5. Fotos PG, Vincent SD, Hellstein JW. Oral candidosis, Clinical, historical, and therapeutic features of 100 cases. Oral Surg Oral Med Oral Pathol. 1992;74(1):41-49.

6. Neville BW. Candidiasis in oral and maxillofacial pathology. Philadelphia: WB Saunders; 2002. p. 189-197.

7. Pankhurst C. Candidiasis oral pharyngeal. Clin Evid. 2005;(13);17011716 .

8. Neville BW. Herpes Simplex Virus Oral and Maxillofacial Pathology. Philadelphia: WB Saunders; 2002. p. 213-220.

9. Porter S. Apthous Ulcers Recurrent. Clin Evid. 2005;(13):1687-1694.

10. Assimakopoulos D, Patrikakos G, Fotika C, Elisaf M. Benign Migratory Glossitis or Geographic Tongue: an enigmatic oral lesion. Am J Med. 2002;113(9):751-755.

11. Heyman WR. Psychotropic agent induced black hairy tongue. Cutis. 2000;(66):25-26.

12. Sarti GM. Black Hairy Tongue. Am Fam Physician. 1990;(41):1751175 .

13. Chan ES. Interventions for treatment of oral lichen planus. Cochrane Database Syst Review. 1999. 